\title{
Docetaxel in combination for advanced gastric cancer
}

\author{
JAFFer A. AJANi \\ University of Texas MD Anderson Cancer Center, 1515 Holcombe Blvd, Houston, Texas, TX 77030-4009, USA
}

\begin{abstract}
Docetaxel is considered to be active in untreated and previously treated patients with gastric carcinoma. In a multinational phase II trial (TAX 325), 158 untreated patients with advanced gastric cancer ( $99 \%$ without prior chemotherapy) were randomized to receive, every 3 weeks, either docetaxel $85 \mathrm{mg} / \mathrm{m}^{2}$ plus cisplatin $75 \mathrm{mg} / \mathrm{m}^{2}$ (TC) or docetaxel $75 \mathrm{mg} / \mathrm{m}^{2}$ plus cisplatin $75 \mathrm{mg} / \mathrm{m}^{2}$, plus a 5-day continuous infusion of $750 \mathrm{mg} / \mathrm{m}^{2}$ 5-fluorouracil (FU; TCF). By intent-to-treat analysis, patients receiving TCF had a significantly higher response rate and longer time to progression. Overall survival in the two arms was not significantly different. Toxicity (particularly gastrointestinal toxicity) was greater with the TCF combination than in the TC arm, and there was a greater need for dose reduction. However, adverse events in both arms were manageable and there were no deaths associated with either regimen. Following these findings, a phase III trial comparing a control arm of cisplatin plus 5-FU against an experimental arm consisting of the TAX 325 phase II docetaxel/cisplatin/5FU regimen is now in progress.
\end{abstract}

\section{Introduction}

While chemotherapy for advanced gastric cancer has been established as superior to best supportive care, no particular single-agent or combination regimen has become accepted as the standard of treatment [1]. Among the single agents that have proven activity in the first-line setting are 5-fluorouracil (5-FU), cisplatin, etoposide, irinotecan, paclitaxel, S-1, and UFT, for which response rates (RRs) ranging from $15 \%$ to $44 \%$ have been reported [2-7]. 5-FU/leucovorin, cisplatin, paclitaxel, and irinotecan have also been used as second-line single agents, with RRs of $17 \%$ to $26 \%$ [8-18]. In addition to the drugs mentioned, docetaxel has ap-

Offprint requests to: J.A. Ajani

Received: April 1, 2002 / Accepted: June 24, 2002 preciable activity in advanced gastric cancer. In three studies of monotherapy in previously untreated patients, docetaxel at a dose of $100 \mathrm{mg} / \mathrm{m}^{2}$ achieved RRs of $17 \%$ to $24 \%$ [17-19]. In the second-line setting, Vanhoefer et al. [20] achieved an RR of 20\% using $100 \mathrm{mg} / \mathrm{m}^{2}$ docetaxel, and Taguchi et al. [21] an RR of $22 \%$ with $60 \mathrm{mg} / \mathrm{m}^{2}$ docetaxel.

Cisplatin is perhaps the most widely used drug in advanced gastric cancer, and two trials have investigated its combination with docetaxel. Roth et al. [22] treated 48 patients with doses of docetaxel ranging from $85 \mathrm{mg} / \mathrm{m}^{2}$ to $100 \mathrm{mg} / \mathrm{m}^{2}$ administered every 3 weeks together with cisplatin $75 \mathrm{mg} / \mathrm{m}^{2}$. Among 45 evaluable patients, the RR was $56 \%$ and median survival, 9 months. Similarly encouraging results were obtained by Ridwelski et al. [23]. In 39 evaluable patients treated with an every-3-week regimen of docetaxel $75 \mathrm{mg} / \mathrm{m}^{2}$ plus cisplatin $75 \mathrm{mg} / \mathrm{m}^{2}$, the RR was $37 \%$ and median survival, 10.4 months.

Given this background, a multinational randomized phase II study (TAX 325) was undertaken to compare the combination of docetaxel with cisplatin against a three-drug arm in which 5-FU was added to docetaxel and cisplatin [24].

TAX 325: docetaxel plus cisplatin vs docetaxel plus cisplatin plus 5-FU

\section{Study design}

TAX 325 involved centers in the United States, Europe, Russia, South America, and Asia. Patients were randomized to one of two treatment arms: docetaxel $85 \mathrm{mg} /$ $\mathrm{m}^{2}$ plus cisplatin $75 \mathrm{mg} / \mathrm{m}^{2}$, with both drugs administered on day 1 every 3 weeks (TC); or docetaxel $75 \mathrm{mg} / \mathrm{m}^{2}$ plus cisplatin $75 \mathrm{mg} / \mathrm{m}^{2}$ on day 1 every 3 weeks plus a continuous infusion of $750 \mathrm{mg} / \mathrm{m}^{2}$-FU given over days $1-5$ (TCF). It should be noted that the $85 \mathrm{mg} / \mathrm{m}^{2}$ docetaxel 
given in the TC arm of the study was a relatively high dose [24].

It was originally intended that the study should accrue around 36 patients in each arm. However, patients continued to be entered into the trial during the period when the initial patients were being assessed. The result was that a total of 158 patients were randomized. With 79 patients entered in each treatment arm, TAX 325 represents one of the largest randomized phase II trials ever undertaken with docetaxel.

The principal efficacy endpoints of the study were response and time to progression. However, data were also collected on overall survival.

Of the 79 patients randomized to TC, 76 were treated, 72 of whom were judged eligible for the study, and 65 ( $82 \%$ of those randomized) were evaluable for response. The corresponding figures for the TCF arm were 79 randomized, 79 treated, 71 eligible, and 64 $(81 \%)$ evaluable for response. The overall rate of ineligibility among all patients entered was $9.5 \%$.

At the time of this report, $5 \%$ of TC patients and $8 \%$ of TCF patients were still on study. The principal reason for discontinuation was disease progression $(50 \%$ of TC and $34 \%$ of TCF patients). Adverse events caused withdrawal of $22 \%$ and $24 \%$ of patients in the two groups.

\section{Patient characteristics}

Table 1 shows the characteristics of the patients treated in the trial. The patients entered were reasonably typical of those in gastric cancer trials, with a median age of 57 years and a good performance status (median, 90\%). Ninety-nine percent of patients had had no prior experience of chemotherapy.

Globally, distal gastric cancer is the most common form of the disease, and this is reflected in the distribution of anatomic sites in this study. Overall, only $32 \%$ of patients had the proximal gastric cancer which is more typical in Western Europe and North America. In 79\% of patients overall, two or more organs were involved. Disease was metastatic in $99 \%$ of cases and locally re-

Table 1. Characteristics of patients treated with docetaxel and cisplatin (TC) or docetaxel plus cisplatin and 5fluorouracil (FU; TCF) [24]

\begin{tabular}{|c|c|c|}
\hline & $\begin{array}{c}\mathrm{TC} \\
(n=76)\end{array}$ & $\begin{array}{c}\text { TCF } \\
(n=79)\end{array}$ \\
\hline Male & $70 \%$ & $77 \%$ \\
\hline Median age & 57 Years & 57 Years \\
\hline Median performance status & $90 \%$ & $90 \%$ \\
\hline Median weight loss & $7 \mathrm{~kg}$ & $7 \mathrm{~kg}$ \\
\hline \multicolumn{3}{|l|}{ Median time from: } \\
\hline Diagnosis to randomization & 2.0 Months & 1.4 Months \\
\hline Last surgery to randomization & 8.8 Months & 8.3 Months \\
\hline
\end{tabular}

current in $1 \%$. Overall, disease was bidimensionally measurable in $79 \%$ of patients.

\section{Results}

The median duration of treatment was 18 weeks in both arms of the study, with a median of five cycles of TC and six cycles of TCF administered. In the TC arm, the relative dose intensity (RDI) of docetaxel was 0.98 , and that of cisplatin, 0.96. Patients in the TCF group also received a high proportion of the intended dose (RDI 0.93 for docetaxel, 0.93 for cisplatin, and 0.95 for 5-FU). In $95 \%$ of TC cycles and $86 \%$ of TCF cycles no dose reduction was required.

Confining the analysis to patients who received treatment according to the study protocol, the RR in the TC group was $35 \%$, and $56 \%$ in the TCF arm. The significantly greater activity of the three-drug regimen was also evident on an intent-to-treat analysis (RR, 28\% with TC and $43 \%$ with TCF). In addition to a higher $\mathrm{RR}$, patients receiving triple therapy experienced a significantly longer time to progression. Although the median overall survival of TC patients was somewhat longer than that in the TCF arm, this difference was not statistically significant.

Grade 4 fever in the absence of infection was reported by $4 \%$ of patients in both arms of the study: there were no grade 3 fevers. The rate of grade $3 / 4$ infection was higher in the TCF than in the TC arm $(10 \%$ vs $4 \%)$. The rates of asthenia were similar in the two arms, while the proportion of patients reporting neurologic events was higher in the two-drug arm, possibly because of the higher dose of docetaxel.

Gastrointestinal toxicities were higher in the TCF arm, and stomatitis (predominantly grade 3 ) occurred only in the group receiving 5-FU. The incidence of grade $3 / 4$ edema was less than $3 \%$ in both arms of the study, as was the incidence of nail changes. Levels of pulmonary toxicity were similarly low $(<3 \%)$ in the two treatment groups. Alopecia affected $4 \%$ of TC and $3 \%$ of TCF patients.

The majority of patients had grade $3 / 4$ neutropenia ( $84 \%$ in both arms). The rates of febrile neutropenia were considerably lower and similar across treatments (16\% with TC and $19 \%$ with TCF). Infection accompanied by grade $3 / 4$ neutropenia was reported in $8 \%$ of TC and $10 \%$ of TCF patients. There were no deaths associated with hematological toxicities.

\section{Discussion}

It is notable that only $1 \%$ of patients included in the study had locally recurrent disease in the absence of metastatic spread. Hence, the study did not contain patients potentially treatable by surgical resection, 
which may, in certain circumstances, bias trial outcome.

The greater toxicity of the three-drug combination was evident in the fact that $14 \%$ of cycles required a dose reduction, while this was true of only $5 \%$ of TC cycles. The excess toxicity attributable to the addition of 5-FU was evident, particularly in stomatitis and diarrhea, which may have been confined largely to the early cycles of treatment, i.e., before dose reductions had been effected.

The somewhat greater toxicity of TCF is accompanied by significantly greater activity - evident in a higher RR and time to progression. The phase II study described was envisaged from the outset as part of an integrated phase II/III program. The protocol called for the better of the two phase II regimens to be compared in a phase III trial against a control arm of cisplatin plus 5-FU. Based on the findings reviewed, the independent data monitoring committee concluded that the TCF regimen should proceed to a randomized phase III trial. In this ongoing study, the comparison is therefore between cisplatin plus 5-FU as the control arm and cisplatin plus 5-FU plus docetaxel. The primary endpoint is time to progression, with overall survival as a secondary endpoint.

Acknowledgments Jaffer Ajani has received honoraria and medical research grants from Aventis Pharmaceuticals Inc.

\section{References}

1. Karpeh MS, Kelsen DP, Tepper JE. Cancer of the stomach. In: DeVita VT, Hellman S, Rosenberg SA, editors. Cancer: principles and practice of oncology. Philadelphia: Lippincott, Williams and Wilkins; 1993. p. 1092-122.

2. Garcia AA, Leichman CG, Lenz HJ, Baranda J, Lujan R, Casagrande Y, et al. Phase II trial of outpatient schedule of paclitaxel in patients with previously untreated metastatic, measurable adenocarcinoma of the stomach. Jpn J Clin Oncol 2001;31:275-8.

3. Ajani JA, Ilson DH, Kelsen DP. Paclitaxel in the treatment of patients with upper gastrointestinal carcinomas. Semin Oncol 1996;23(5 Suppl 12):55-8.

4. Futatsuki K, Wakui A, Nakao I, Sakata Y, Kambe M, Shimada Y, et al. Late phase II study of irinotecan hydrochloride (CPT-11) in advanced gastric cancer. CPT-11 Gastrointestinal Cancer Study Group. Gan To Kagaku Ryoho (Jpn J Cancer Chemother) 1994; 21:1033-8.

5. Armand JP, Cunningham D, van Cutsem E, Misset JL, Kohne $\mathrm{CH}$. Clinical advances with topoisomerase I inhibitors in gastrointestinal malignancies. Anticancer Drugs 1999;10(Suppl 1): S5-12.

6. Kohne CH, Wils JA, Wilke HJ. Developments in the treatment of gastric cancer in Europe. Oncology (Huntingt) 2000;14(12 Suppl 14):22-5.

7. Tahara M, Ohtsu A. Latest progress in chemotherapy for advanced gastric cancer. Gan To Kagaku Ryoho (Jpn J Cancer Chemother) 2000;27:2048-58.
8. Bokemeyer C, Hartmann JT, Lampe CS, Clemens MR, Quietzsch D, Forkmann L, et al. Paclitaxel and weekly 24-hour infusion of 5-fluorouracil/folinic acid in advanced gastric cancer. Semin Oncol 1997;24(6 Suppl 19):S19-96-S19-100.

9. Kim YH, Shin SW, Kim BS, Kim JH, Kim JG, Mok YJ, et al. Paclitaxel, 5-fluorouracil, and cisplatin combination chemotherapy for the treatment of advanced gastric carcinoma. Cancer 1999;85:295-301.

10. Murad AM, Petroianu A, Guimaraes RC, Aragao BC, Cabral LO, Scalabrini-Neco AO. Phase II trial of the combination of paclitaxel and 5-fluorouracil in the treatment of advanced gastric cancer: a novel, safe, and effective regimen. Am J Clin Oncol 1999;22:580-6.

11. Shirao K, Shimada Y, Kondo H, Saito D, Yamao T, Ono H, et al. Phase I-II study of irinotecan hydrochloride combined with cisplatin in patients with advanced gastric cancer. J Clin Oncol 1997;15:921-7.

12. Sato A, Kurihara M, Matsukawa M, Shimada K, Yamazaki T, Nakamachi M, et al. Preliminary study of fortnightly irinotecan hydrochloride plus cisplatin therapy in patients with advanced gastric and colorectal cancer. Cancer Chemother Pharmacol 2001; 47:380-4.

13. Ajani JA, Baker J, Pisters PW, Ho L, Feig B, Mansfield PF. Irinotecan plus cisplatin in advanced gastric or gastroesophageal junction carcinoma. Oncology (Huntingt) 2001;15(3 Suppl 5):524.

14. Pozzo C, Bugat R, Peschel C, Gorbunova V, Valvere V, Zaluski J, et al. Irinotecan in combination with CDDP or 5-FU and folinic acid is active in patients with advanced gastric or gastro-oesophageal junction adenocarcinoma: final results of a randomised phase II study (abstract). Proc Am Soc Clin Oncol 2001;20:531.

15. Grau JJ, Martín M, Gascón P, Pera M, Graupera J, Balcells M, et al. Phase II study of irinotecan (CPT-11) and mitomycin c (MMC) combination in patients with advanced gastric cancer (ENG). Preliminary results (abstract). Proc Am Soc Clin Oncol 2001; 20:2284.

16. Findlay PN, Ackland S, Gebski V, Yuen J, Boyer M, Walpole E, et al. Phase II study of irinotecan, leucovorin and 5FU (ILF) in advanced gastric cancer (abstract). Proc Am Soc Clin Oncol 2001;20:655.

17. Sulkes A, Smyth J, Sessa C, Dirix LY, Vermorken JB, Kaye S, et al. Docetaxel (Taxotere) in advanced gastric cancer: results of a phase II clinical trial. EORTC Early Clinical Trials Group. Br J Cancer 1994;70:380-3.

18. Einzig AI, Neuberg D, Remick SC, Karp DD, O'Dwyer PJ, Stewart JA, et al. Phase II trial of docetaxel (Taxotere) in patients with adenocarcinoma of the upper gastrointestinal tract previously untreated with cytotoxic chemotherapy: the Eastern Cooperative Oncology Group (ECOG) results of protocol E1293. Med Oncol 1996;13:87-93.

19. Mavroudis D, Kourousis C, Androulakis N, Kalbakis K, Agelaki $\mathrm{S}$, Kakolyris S, et al. Frontline treatment of advanced gastric cancer with docetaxel and granulocyte colony-stimulating factor (G-CSF): a phase II trial. Am J Clin Oncol 2000;23:341-4.

20. Vanhoefer U, Wilke H, Harstrick A, Kalbakis K, Agelaki S, Kakolyris S, et al. Phase II study of docetaxel as second line chemotherapy (CT) in metastatic gastric cancer (abstract). Proc Am Soc Clin Oncol 1999;18:1163.

21. Taguchi T, Sakata Y, Kanamaru R, Fazio N, Aapro MS, Pagani O, et al. Late phase II clinical study of RP56976 (docetaxel) in patients with advanced/recurrent gastric cancer: a Japanese Cooperative Study Group trial (group A). Gan To Kagaku Ryoho (Jpn J Cancer Chemother) 1998;25:1915-24.

22. Roth AD, Maibach R, Martinelli G, Fazio N, Aapro MS, Pagani O, et al. Docetaxel (Taxotere)-cisplatin (TC): an effective drug combination in gastric carcinoma. Swiss Group for Clinical Cancer Research (SAKK), and the European Institute of Oncology (EIO). Ann Oncol 2000;11:301-6. 
23. Ridwelski K, Gebauer T, Fahlke J, Kroning H, Kettner E, Meyer F, et al. Combination chemotherapy with docetaxel and cisplatin for locally advanced and metastatic gastric cancer. Ann Oncol 2001;12:47-51.

24. Ajani JA, Fodor M, Van Cutsem E, Tjulandin S, Moiseyenko V,
Cabral F, et al. Multinational randomized phase II trial of docetaxel (T) and cisplatin (C) with or without 5-fluorouracil (FU) in patients (pts) with advanced gastric or GE junction adenocarcinoma (AGC-AGEJC) (abstract). Proc Am Soc Clin Oncol 2000;19:957. 